\title{
Actividades económicas locales y regionales desarrolladas por las elites en Santiago del Estero. El caso de Alfaro y sus descendientes durante 1700-1750.
}

\section{Local and regional economical activities developed by the elite in Santiago del Estero. The Alfaro and his descendants case during 1700-1750.}

\author{
Héctor F. Peralta Puy \\ Universidad Nacional de Santiago del Estero, Argentina. \\ Contacto: hectorperaltapuy@gmail.com
}

\section{Resumen}

El presente trabajo de investigación posee sus inicios a partir de lecturas que fueron incrementando una inquietud que posee nombre y apellido: Alonso de Alfaro. Entre las bibliografías escritas por diversos autores santiagueños, existen referencias acerca de la importancia política, militar y religiosa que tuvo Alfaro en relación a sus actuaciones desarrolladas en el cabildo durante las primeras décadas del siglo XVIII. Sin embargo, las fuentes consultadas no poseen investigaciones relacionadas a las actividades económicas desempeñadas por Alfaro, algo que evidentemente fue una constante en la región debido a que un personaje destacado en la política y en las armas también era un comerciante fletador o un inversionista inmobiliario. Por estas cuestiones, trataremos de responder al siguiente interrogante: ¿cuál fue la importancia económica que tuvo Alfaro en Santiago del Estero y en el resto de las ciudades integrantes de la región del Tucumán?

Una vez que estuvieron agotados los libros con referencias políticas al teniente de gobernador y mientras avanzábamos búsqueda de la información en el Archivo Histórico y en la Dirección General de Catastro (ambos organismos situados en Santiago del Estero), las reseñas hacia Alfaro se fueron direccionando desde las mencionadas actividades hacia el comercio local y regional y a las inversiones inmobiliarias rurales y urbanas efectuadas 
por él mismo o por la red familiar que fue confeccionada de acuerdo a los casamientos estratégicos de sus hijas y su nieta.

Palabras claves: Alfaro; Red familiar; Camino del Palomar; inversiones; comerciantes.

\section{Abstract}

This research work has its beginnings from readings that were increasing a concern that has a name and surname: Alonso de Alfaro. Among the bibliographies written by various authors from Santiago, there are references to the political, military and religious importance that Alfaro had in relation to his actions developed in the town hall during the first decades of the eighteenth century.However, the sources consulted do not have research related to the economic activities carried out by Alfaro, something that was clearly a constant in the region because a prominent character in politics and weapons was also a charterer or real estate investor. For these questions, we will try to answer the following question: what was the economic importance that Alfaro had in Santiago del Estero and in the rest of the cities that make up the Tucumán region?

Once the books with political references to the lieutenant of the governor were exhausted and while we were searching for information in the Historical Archive and in the General Directorate of Cadastre (both agencies located in Santiago del Estero), the reviews towards Alfaro were directed from the aforementioned activities towards local and regional commerce and rural and urban real estate investments carried out by him or by the family network that was made according to the strategic marriages of his daughters and granddaughter.

Keywords: Alfaro; Family Network; Palomar road; Inversions; Merchants.

\section{1- Introducción}

El presente trabajo de investigación posee sus inicios a partir de diversas lecturas que fueron incrementando una inquietud representada en don Alonso de Alfaro. Este inmigrante fue un personaje destacado de la época colonial en Santiago del Estero y en las ciudades del Tucumán.

Entre sus numerosas actividades desarrolladas, fue sargento mayor y maestre de campo y además el hombre de confianza en la región -durante muchos años- del gobernador Urizar y Arespacochaga. Con el transcurso del tiempo, Alfaro desempeñó en Santiago del Estero los cargos administrativos de teniente de gobernador, justicia mayor y capitán a guerra, gobernador interino y alférez real. En relación a los descendientes de los primitivos habitantes, fue encomendero del pueblo de Guañagasta en 1702, depositario de 
los indios Guaype en 1707, participante de las campañas militares regionales iniciadas en 1710 y encargado de elaborar los padrones de los pueblos de indios en 1714 y 1721 . Sus ocupaciones religiosas lo llevaron a ser un activo colaborador y benefactor de los religiosos franciscanos, domínicos y jesuitas, por lo que hizo refaccionar con su propio peculio la Iglesia Matriz y el Templo de la Merced.

Como podemos observar, existen nutridas referencias acerca de la importancia política, militar y religiosa que tuvo Alfaro en relación a sus actuaciones desarrolladas en el cabildo durante las primeras dos décadas del siglo XVIII. Sin embargo, las distintas fuentes bibliográficas consultadas no poseen informaciones relacionadas a las actividades económicas desempeñadas por Alfaro, algo que evidentemente fue una constante en la región debido a que un personaje destacado en la política y en las armas, generalmente también era un comerciante fletador o un inversionista inmobiliario con estancias y animales. Por estas cuestiones, trataremos de responder al siguiente interrogante que fue diagramado como uno de los principales objetivos de la investigación: ¿cuál fue la importancia económica que tuvo don Alfaro en Santiago del Estero y en el resto de las ciudades integrantes de la diversa región del Tucumán?

Una vez que estuvieron agotados los libros con referencias políticas al teniente de gobernador y mientras avanzábamos cada vez más en la exhaustiva búsqueda de la información en el Archivo Histórico y en la Dirección General de Catastro (ambos organismos gubernamentales situados en Santiago del Estero), las reseñas hacia Alfaro se fueron direccionando desde las mencionadas actuaciones políticas hacia las actividades de las armas pero principalmente hacia el comercio local y regional y a las inversiones inmobiliarias rurales y urbanas efectuadas por él mismo o por la red familiar confeccionada de acuerdo a los casamientos estratégicos de sus dos hijas y su nieta. Entonces, a través de los documentos coloniales consultados, pudimos diagramar un Estudio de Caso cuyos variados objetivos se fundamentan en la indagación de las actividades militares, comerciales (locales y regionales) y políticas conjuntamente con las inversiones inmobiliarias (rurales y urbanas) realizadas por Alfaro y la elite integrante de su red familiar en Santiago del Estero y en la región del Tucumán durante los años 1700 y 1753.

La reconstrucción de las actividades comerciales de la red familiar fue posible por los documentos coloniales de las secciones Tribunales y Asuntos Generales del Archivo Histórico, aunque también se destaca en el proceso el Archivo Gráfico de la Dirección General de Catastro. Estas fuentes fueron complementadas con las publicaciones de la Revista del Archivo y con los libros capitulares seleccionados por Ángel Justiniano Carranza en 1882, además de lo realizado por la Academia Nacional de la Historia en cuanto a los documentos del cabildo santiagueño. 
Por todas estas razones y los objetivos detallados, utilizaremos una interdisciplinariedad social basada en los análisis cualitativos y cuantitativos debido a la complejidad del tema en cuestión, el cual se encuentra representado por la economía y la política como fundamentos de los estudios históricos.

\section{2-}

Los caminos coloniales en el actual norte argentino se conformaron conjuntamente con el establecimiento de las ciudades en la segunda mitad del siglo XVI,(1) por lo que la utilización de estas vías conectoras fue aumentando mientras el comercio y las relaciones humanas con el Alto Perú se hicieron cada vez más intensos, destacándose los derroteros del Palomar, el Real y de La Plata, los cuales tuvieron una mayor circulación de carretas (cada uno en determinado momento y contexto miliar, social y judicial y según las circunstancias y necesidades de los viajeros) debido a los alzamientos de los Calchaquíes producidos en las tierras altas (1560-1563)(2) que pusieron en peligro a las recientes poblaciones allí establecidas y al antiguo Camino del Inca cercano a las mismas, siendo este derrotero el primero por el que entraron los conquistadores al Tucumán. Hacia finales del siglo XVII comenzaron los ataques desde el oriente de la región, más precisamente por las acciones de los Mocovíes provenientes desde el Chaco Gualamba hacia la entrada ubicada en Esteco, por lo que fueron necesarias las acciones defensivas y ofensivas por parte de los sucesivos gobernadores del Tucumán.(3)

En ese contexto militar a nivel regional -en donde los avances de los Mocovíes repercutieron negativamente en las economías agrarias de las fronteras de las ciudades por los peligros a los que se veían expuestas las haciendas y las vías comunicacionales dirigidas hacia el Alto Perú- resaltaba como comerciante en Santiago del Estero don Alonso de Alfaro.(4) Entre sus primeras actividades desarrolladas desde 1700 en la Madre de Ciudades, destacamos una mediación asumida en un conflicto entre dos tratantes y por la cual le otorgaron un poder para cobrar lo adeudado por uno de ellos;(5) aunque el trabajo como fletador fue uno de los más importantes oficios que tuvo desde sus inicios como comerciante, concertándose en 1702 el traslado con don Francisco Núñez de Ávila de unas 13.079 cabezas de ganado vacuno hasta Jujuy, comprometiéndose don Alonso a entregar los animales al capitán González de Castilla.(6) De esta manera, dio comienzo para Alfaro la construcción de su fortuna personal en base a sus primeras actuaciones mercantiles en Santiago del Estero y en la región del Tucumán.

Las actividades y el prestigio de comerciante regional de Alfaro comenzaron a afianzarse desde 1703, año en el que compadeció conjuntamente con el mercader tratante Simón de Larramendi ante el cabildante y estanciero José López de Velazco por un convenio sobre fletamentos de carretones con ropa de la tierra y Géneros de Castilla para 
trasladar hacia la ciudad de Santa Fe. El arreglo fue por la suma de $\$ 4.583$ y 4 reales, concertándose además el transporte de las mercaderías hacia Jujuy, por lo que también involucraron en el contrato el pago de los soldados para la seguridad de los productos (7) Estas escoltas eran vitales para que las cargan llegasen a destino debido a que los caminos hacia el norte eran inseguros, ya sea si optaban por el Camino Real o por el Palomar (el Camino de La Plata ya había entrado en decadencia).

Por estas razones, la nueva autoridad que asumió el mando en 1707 dedicó sus esfuerzos para que los caminos comerciales, las ciudades y las estancias fronterizas de la región tuviesen seguridad, por lo que una de las primeras gestiones de Urizar y Arespacochaga fue recorrer la jurisdicción del Tucumán y elaborar un informe acerca del estado de las fronteras, el cual tuvo como conclusión que los ataques despoblaron las estancias ganaderas y los establecimientos agrícolas, dejando como consecuencia a las ciudades expuestas y vulnerables, por lo que propuso a los tenientes de gobernadores el renunciamiento momentáneo de la guerra defensiva y el comienzo de una serie de ataques hacia el interior del Chaco (siendo efectuados los mismos desde 1710 con resultados positivos aunque provisorios).

Una vez que estuvieron realizadas las acciones ofensivas con participaciones de Alfaro y de soldados y pertrechos devenidos desde la Madre de Ciudades, se dio inicio a una nueva etapa de poblamiento y de refuerzos en las fronteras regionales para el resguardo de los caminos comerciales y las estancias (algo en lo que los comerciantes como Alfaro se encontraban interesados en lograr, asegurándose de esa manera las actividades mercantiles), por lo que se instalaron fuertes y sectores vigilados desde Esteco conjuntamente con poblaciones rurales permanentes por intermedio de la reactivación de los establecimientos agrícolas y ganaderos a partir de la primera década del siglo XVIII.

Entonces, una vez aseguradas -momentáneamente- las fronteras y los caminos conectores, Alfaro se propuso complementar y acrecentar sus actividades comerciales con la tenencia y explotación de estancias ubicadas en las fronteras de las jurisdicciones, por lo que solicitó al gobernador el 9 de enero de 1713 una merced de tierras en "...Tenené...tomando por centro todos los ojos de agua que tiene, y tres leguas de contorno, el cual dicho paraje a más de treinta años que está desierto y despoblado y ha estado habitado del enemigo, y así mismo el paraje del Palomar contiguo al antecedente tirando siempre al norte, y que el centro sea la aguada de dicho Palomar...".(8) Observamos en el pedido que ambas estancias (9) poseían aguas y contornos de tres y dos leguas respectivamente, siendo importante para el solicitante el hecho de que estuvieran despobladas como consecuencias de sus cercanías a los lugares por donde incursionaron los naturales en los años anteriores a la ofensiva de 1710. Esas propiedades se encontraban situadas a 20 leguas rectas de la ciudad de Santiago por el Camino del 
Palomar (actual departamento Jiménez) que llegaba hacia Jujuy, siendo ésta una alternativa para acortar el recorrido hacia Charcas en lugar de seguir el paso por San Miguel a través del Camino Real, por lo que se fue creando una situación que representaba una ventaja para los comerciantes carreteros y para Alfaro como nuevo propietario de las estancias.

La aprobación de la merced por parte de Urizar se justificaba por la política emanada desde la gobernación de erradicar los espacios fronterizos despoblados para evitar nuevas incursiones de los Mocovíes; además, tuvo influencia en la decisión la participación del solicitante en la ofensiva iniciada en 1710 en la cual había gastado $\$ 14.000$ de su propio peculio en pagos a soldados y demás gastos generales (armas, municiones, bastimentos, etc.). Por otro lado, el acceso a las tierras significaba para Alfaro una estrategia política y económica para recuperar el mencionado dinero y a la vez una importante inversión en bienes raíces rurales.

Entonces, el Tercer Período de Propietarios (10) de las estancias dio inicio con las posesiones ejecutadas el 30 de septiembre y el 2 de octubre de 1713, encargándose el maestre de campo Francisco de Luna y Cárdenas de implementar los preceptos legales castellanos, los cuales consistían en tomar de la mano a Salvatierra (representante de Alfaro) y pasarla por las aguadas o las plantas (arrancando yerbas) y así efectuar la posesión formal de los títulos "...actual, real, corporal e jure domine vel quasi...". Finalmente, el 9 de octubre de 1714, fueron comprados por Alfaro los derechos de los establecimientos a los domínicos en \$200.(11) A partir de esos momentos, fueron reconstruidas las estancias y utilizados los campos de Tenené para la cría de ganados varios y para servir como lugar de aprovisionamiento de agua para los animales de los comerciantes y viajeros. Por otro lado, los terrenos del Palomar fueron acondicionados para vivienda y habitación, aunque también se introdujeron en ellos diversos animales.

De acuerdo a estos acontecimientos, los objetivos políticos, económicos y sociales de Alfaro diagramados en torno a sus estancias fueron: recuperar los $\$ 14.000$ destinados a la pacificación de las fronteras que aseguraron los límites de las jurisdicciones y los caminos rurales; reactivar el Camino del Palomar;(12) poblar la zona fronteriza con trabajadores, peones, agregados y fletadores; acrecentar su patrimonio personal mediante las inversiones inmobiliarias rurales; efectuar actividades comerciales con los carreteros (fletamentos de carretas, intercambio o ventas de animales de carga, ventas de bastimentos -productos agrícolas o alimentos- y lugar de descanso para los carreteros 0 viajeros); brindar seguridad a los comerciantes por intermedio de guardias o escoltas y conservar y acrecentar los bienes adquiridos mediante una red familiar consolidada a través de los matrimonios de sus dos hijas. 
Con el transcurso de los años, más precisamente en 1717, las actividades mercantiles de Alfaro se diversificaron hacia las ventas de mercaderías en tierras lejanas a través de poderes otorgados a terceros, tal como hizo con el mercader tratante Pedro Núñez de Herrera “...para que en la Villa Imperial de Potosí o en otra cualquier ciudad, villa o lugar pueda hacer trato de las mercaderías y géneros que le parecieren y en la cantidad o cantidades que gustase y pueda...".(13) En 1719, fueron otorgadas por Alfaro diversas representaciones legales para que en su nombre puedan demandar y cobrar los dineros adeudados por los comerciantes de las regiones alejadas, siendo el capitán don Bernardo de Ochoa Montaos el encargado de ejecutar esa función en las "...Provincias del Perú.....(14)

Las ocupaciones de comerciante, vendedor y estanciero, le posibilitaron a Alfaro en 1723 la adquisición por $\$ 70$ de otra destacada propiedad, aunque esta vez ubicada en la ciudad, más precisamente al frente de la plaza pública. Esta transacción fue notificada por el alcalde de primer voto José Díaz Caballero, siendo el inmueble una parte de las pertenencias del convento de San Francisco, por lo que fue solicitado al síndico propietario José López de Velazco para que sea el representante de la institución religiosa ante el comprador. Este solar estaba despoblado y se encontraba “...contiguo a las casas del cabildo y fosa real por la parte del poniente y por la del naciente sin población inmediata, y por la del sur linda con casa y morada del Capitán Juan de Paz y Figueroa y por la del norte con una laguna grande haciendo esquina y libre la calle con dichas casas del cabildo...".(15)

Por todas estas injerencias en el comercio, en las inversiones inmobiliarias y en los asuntos públicos santiagueños y regionales, Alfaro fue solicitado para las ejecuciones de deudas por parte de comerciantes residentes en Chile (16) y Salta (17) entre 1724 y 1725 (respectivamente), los cuales mantuvieron negocios y contratos de fletes con moradores de Santiago del Estero.

Finalmente, luego de haber desarrollado durante años la tenencia del gobierno de la ciudad en forma paralela con los negocios comerciales e inmobiliarios, Alfaro pudo desempeñar la función administrativa y militar más importante de la región, es decir, ocupar el cargo de gobernador del Tucumán, aunque fue por poco tiempo y de manera interina.

La muerte de Alfaro se produjo en 1726 luego de padecer una enfermedad durante un tiempo, por lo que antes del deceso fueron declaradas sus hijas Isabel y Josefa como herederas (su esposa Manuela ya había fallecido). Un año después comenzaron a aparecer acreedores que reclamaron los pagos de las deudas contraídas por Alfaro durante el desarrollo de sus actividades comerciales. Tal es así que en 1727, don José de Villa y Burgos hizo un requerimiento por el pago de $\$ 145$.(18) En 1733, Herrera y Loziaga 
y su padre Antonio José de Herrera, solicitaron los pagos de $\$ 3.490$ y 4 reales y $\$ 2.797$ con 4 y 1/2 reales,(19) respectivamente. Por último, las casas comerciales de Buenos Aires hicieron un reclamo por $\$ 30.977$.(20) Estas cuatro obligaciones recayeron sobre el sobrino de Alfaro, el maestre de campo Antonio Bozán de Alfaro.

Las hijas de Alfaro y de Manuela de Alba Bravo de Zamora, Josefa e Isabel, contrajeron sus nupcias cuando su padre todavía estaba con vida, y una vez fallecido, consolidaron la red social diagramada por su padre de acuerdo a las conveniencias sociales.(21) Josefa contrajo matrimonio con el capitán Domingo Gerónimo de Frías (22) e Isabel con el maestre de campo Gerónimo de Peñaloza.(23)

Por intermedio de estos enlaces fueron transferidas las propiedades y la distribución del poder político y económico, es decir, fue permitida por Alfaro las participaciones de sus yernos en los negocios familiares mientras éstos contribuyeron a conservar las jerarquías sociales y políticas. Con la creación de la red familiar, el primer liderazgo visible se encontraba en Alfaro-Bravo de Zamora (aunque no vimos ninguna acción económica ni política por parte de doña Manuela), mientras que luego de 1726 la dirección fue recayendo en Isabel y Antonio Bozán, hija y sobrino de Alfaro. Esta red también mantuvo activas negociaciones entre sus propios miembros, por lo que no se registraron conflictos de intereses durante los primeros años aunque eso no tardaría mucho tiempo en modificarse en un grupo conformado por motivaciones personales con características comerciales y financieras.

Las familias de la elite utilizaban de manera regular el vínculo del mayorazgo castellano al momento de legar sus bienes con la intensión de que éstos no se pierdan entre todos los posibles herederos, por lo que se dejaba en el primer hijo la mayor parte de las propiedades adquiridas para aumentar los bienes con el transcurso del tiempo. Al respecto, Ana María Presta se refiere a la figura del mayorazgo como una elección por parte de la elite para que las adquisiciones permanezcan dentro del linaje a pesar de que "...la ley castellana otorgaba a los nacidos del legítimo matrimonio iguales derechos de herencia, muchas familias se ampararon en los espacios que dejaba la ley y favorecieron a uno de ellos, a quien donaban, generalmente, las partes de libre disposición y mejora con que podían aumentar la cuota obligatoria o legitima que correspondía a todo heredero...los que habían acumulado un patrimonio considerable y temían que se licuara en una generación al distribuirse entre los numerosos herederos legítimos, concentraban tierra y derechos -sobre los que pesaba la prohibición de enajenación- en uno de los hijos, el mayor varón, que se beneficiaba por el azar biológico...". Pero, ¿qué sucedía en los casos como Alfaro al haber engendrado dos hijas?

En el poder para testar, fue declarado por Alfaro que sus dos hijas eran las herederas legítimas, lo que nos hace pensar en una posible distribución equitativa de los bienes, 
aunque según los registros fue heredada por Josefa la encomienda de Guañagasta mientras que Isabel adquirió las estancias de Tenené y Palomar conjuntamente con la chacra número 70 que se alimentaba de la acequia real y el solar contiguo a las casas del cabildo. Existen dos posibilidades para explicar la herencia: estuvo presente la figura del mayorazgo para favorecer al matrimonio de Isabel o tuvo que haber influido en Alfaro la mejor situación económica de Josefa y su esposo con respecto a la tenencia de propiedades y recursos (Frías concentraba la merced de Tipiro y las administraciones de los pueblos de Manogasta y Sumamao).

El primer ejemplo de las negociaciones de la red familiar con terceros lo encontramos en un otorgamiento de poderes de venta realizado en 1725 por parte de Frías hacia Antonio Bozán de Alfaro para la transacción de una mulata criolla llamada María, quien contaba por ese entonces con 19 años. Esta operación fue realizada con don José de Espeche -vecino de San Fernando del Valle de Catamarca- por la suma de \$405.(24) Dos años después, nuevamente fue otorgado por Frías otro poder a Bozán de Alfaro, aunque esta vez para cobrar o recaudar los montos adeudados.(25)

En 1731 hizo su ingreso en el círculo social de la familia el capitán Esteban de Urrejola -inmigrante proveniente de la Villa de Ochandiano, Vizcaya, una de las tres provincias del País Vasco (Urrejola Montenegro: 2010)-, quien contrajo matrimonio con doña Josefa de Peñaloza, hija de Gerónimo e Isabel y hermana de Félix Ignacio (casado con doña María Francisca Ibarra). Con la adhesión del joven vasco se produjo la continuidad de las uniones estratégicas entre las hijas de los políticos con los comerciantes destacados.(26) Para otorgar una gracia económica a la nueva pareja, Isabel y Gerónimo realizaron una carta dotal en 1731(27) por la cual beneficiaron al enlace de su hija con la finca del Palomar valuada en $\$ 2.000$ (el importe refleja el valor del camino y su reactivación económica representada por sus actividades ganaderas). Este instrumento legal castellano se consideraba como un adelanto de la herencia y en este caso fue útil para conservar el patrimonio familiar, además de ser un indicador de la posición económica de la familia Alfaro-Bravo de Zamora y su línea Peñaloza-Alfaro, posibilitándose de esta manera el inicio de un nuevo patrimonio a través de una inversión en la rama Urrejola-Peñaloza. Los aportes de la familia Urrejola al matrimonio fueron $\$ 1.000$ mientras que la carta dotal totalizaba $\$ 34.255$. Estos adelantos en la herencia (28) beneficiaron al matrimonio de Josefa y relegaron económicamente a su hermano Félix Ignacio.

Mientras tanto, los negocios continuaban produciéndose en el seno de la familia, no solamente contrayéndose y generándose activos sino también obligaciones a pagar por parte de los integrantes de la red familiar, constando diversos compromisos a través de empréstitos por los cuales firmaron algunos pagarés, siendo un ejemplo de ello el 
documento de retribución de deuda firmado en 1731 por Gerónimo de Peñaloza y Bozán de Alfaro a favor de Francisco Antonio de Olleta, el cual había entregado en calidad de préstamo la suma de $\$ 1.887$ y 5 reales en plata.(29) En cuanto a Frías, en 1733 fue deudor por $\$ 62$ de la Real Caja con respecto a la administración del pueblo de indios de Manogasta perteneciente a la corona española.(30)

Pero, existieron una cantidad mayor de cobros de deudas a terceros que salidas de activos o dineros como partes de pagos, estando Isabel como partícipe en estos negocios. Esto se encuentra representado en 1732 cuando fue solicitado por Peñaloza -en representación de su esposa- el pago de \$1.500 a Gregorio Riberos.(31) En 1733 fue generada una situación más delicada cuando a través de Bozán de Alfaro, Isabel solicitó el embargo de los bienes de la viuda del sargento Bernabé Pereyra por una obligación de $\$ 327$ con 7 reales. En esa oportunidad, Isabel requirió la entrega de la vivienda en donde moraba la demandada con sus hijos, por lo que tuvo que intervenir el defensor de menores.(32)

Los cobros de deudas con ayuda de familiares fueron una modalidad recurrente a través de los años, por ejemplo en la entrega de un poder judicial en 1733 por parte de don Gerónimo de Peñaloza a su yerno Urrejola para la percepción de lo adeudado a través de “...plata, joyas, mercaderías y otros cualesquiera géneros y especies.....(33) Otra operación fue realizada por Urrejola en 1747, aunque esta vez con Félix Ignacio de Peñaloza -cuñado-, quienes firmaron a favor del primero por un préstamo de 317 libras de cera negra del Salado.(34) La participación más importante de Urrejola en la red familiar fue realizada en 1732 a través de una operación en donde se incluyeron los bienes ganaderos de la estancia del Palomar, por lo que fueron intercambiadas en febrero -con el mercader tratante Martín de Sarría- unas 2.000 cabezas de ganado vacuno a $\$ 3.5$ cada ejemplar, haciéndose un total de $\$ 7.000$ representados en 5.000 varas de ropa de la tierra a 8 reales cada vara y 2.000 columnas de 8 reales cada una.(35)

Mientras se desarrollaban las actividades comerciales de la red familiar, comenzaron a surgir conflictos internos por los intereses que cada uno personificaba, es decir, cada representante de las cuatro familias (Peñaloza, Frías, Urrejola y Bozán de Alfaro) poseía y aspiraba a un beneficio personal por sobre el grupo económico.

El primer problema fue generado en 1732 entre los yernos de Alfaro debido a los activos de las estancias escalonadas en el Camino del Palomar. Las funciones principales que tuvieron estos establecimientos fueron la cría de ganado, el comercio de los productos agrícolas de la zona (ventas de bastimentos), los fletamentos de carretas, el alquiler o el intercambio de animales de carga con los carreteros y la acogida para el descanso de los conductores y arrieros. El Palomar tuvo bienes importantes para el desarrollo de estas actividades, por lo que se destacaron los 50 bueyes como animales de tracción en el 
proceso de la agricultura o en la conducción de los carretones ( $\$ 300, \$ 6$ cada uno), las 2.000 cabezas de ganado que pastaban a campo abierto ( $\$ 8.000, \$ 4$ cada una), las 50 mulas utilizadas para los envíos ( $\$ 1.200, \$ 24$ cada una) y las 2 carretas y los 2 carretones para las cargas y los transportes, entre otros activos; mientras que en 1730, Tenené albergó 1.333 cabezas de ganado vacuno (\$3.999, \$3 cada una). Entonces, alrededor de estos activos fue desarrollándose la administración del lugar por parte de Frías y Peñaloza luego de la muerte de Alfaro, siendo prueba de ello el ganado de ambos establecimientos que fue intercambiado de manera forzosa en junio de 1732 por Frías cuando hizo la entrega de \$3.999 a Peñaloza por las 1.333 cabezas de ganado de Tenené. Esta transacción fue realizada para evitar problemas mayores e incluso se dio previo aviso al cabildo como una manera para que se encuentre prevenido de la operación desarrollada en el Camino del Palomar.(36)

Por otro lado, mientras pasaron los años se fueron intensificando las incursiones de los Mocovíes por las zonas fronterizas entre los territorios de San Miguel, Santiago del Estero y Salta, lo que fue afectando al tránsito de las carretas por el Camino del Palomar y por el Camino Real, por lo que se sintieron los efectos negativos en las estancias que se encontraban en la zona norte santiagueña cercana a los límites con la jurisdicción de San Miguel. Por estas cuestiones, la red familiar fue decayendo con sus actividades comerciales en la década de 1730 (por lo que llegaron incluso a vender el ganado vacuno que pastaba por Tenené y Palomar, según vimos en el párrafo anterior).

Además, fue tomando importancia la desaparición física de los integrantes de la red familiar, tal como fue el caso de Gerónimo de Peñaloza. Con este deceso se fue despertando un viejo recelo suscitado por la carta dotal que había favorido al enlace de Josefa con Urrejola, por lo que fue reclamado por Félix Ignacio en 1750 la parte de la herencia que le correspondía según los alegatos labrados en los documentos judiciales del cabildo, aduciéndose en el expediente en cuestión que todos los bienes "...quedaron en poder de mi madre...a que soy legítimo heredero con doña Josefa...y al tiempo de contraer matrimonio...se le dieron en dote más de \$35.000 no correspondientes al cúmulo de bienes....no pudiendo por entonces decidir mi derecho...(ya que los bienes heredados por mi padre fueron)...disipados la mayor parte por don Esteban de Urrejola..... La respuesta del denunciado fue argumentar que no fue su tutor luego de la muerte de Peñaloza y que no era digna la demanda porque de la carta dotal no fueron recibidos todos los bienes detallados. Por esto, llegaron entonces a un juicio en donde la influencia de Isabel se puso de parte de su hijo.(37)

Las realidades del decaimiento de las estancias por los peligros en las fronteras, el ocaso del grupo por los fallecimientos de sus integrantes, la lógica disminución de las actividades comerciales y las disputas familiares por los bienes, aceleraron la finalización 
del grupo económico y político, aunque el proceso tuvo su final cuando doña Isabel comenzó a vender las propiedades rurales y urbanas desde la década de 1750, como la estancia de Tenené en 1753 por el valor de $\$ 3.000$, la chacra número 70 de la acequia real y el solar que se encontraba contiguo a las casas del cabildo valuado en $\$ 70$, siendo adquirido este último por don Juan de Salvatierra.

Entonces, de los 25 movimientos consignados por la red familiar diagramada por don Alonso, Antonio Bozán participó en 4 actividades conjuntamente con Frías (1725 y 1727), Peñaloza (1731) e Isabel (1733). En cuanto a tres los matrimonios, Isabel con Peñaloza tuvieron una mayor intervención en el grupo con 12 actividades económicas (48\%), mientras que Josefa con Frías tuvieron 8 (32\%) y la unión de Urrejola con Josefa alcanzó un número de 5 participaciones (20\%).

\section{3- Conclusiones}

Alonso de Alfaro fue uno de los comerciantes más importantes de la región desde el inicio del siglo XVIII hasta 1726, lo que lo convirtió en un destacado integrante de la elite militar, política y terrateniente de Santiago del Estero. Tuvo entre sus bienes importantes estancias rurales y propiedades urbanas, las cuales fueron legadas a sus hijas como un destacado capital real o material e incluso como un patrimonio inmaterial representado en el capital simbólico de su apellido y de su posicionamiento social logrado a través de sus actividades de mediador, comerciante de géneros de la tierra y de Castilla, fletador, estanciero, inversionista rural y propietario de diversos inmuebles en la ciudad. Alfaro, con sus acciones comerciales, militares y políticas y con sus adquisiciones en el Camino del Palomar, pudo ejercer su influencia en el desenvolvimiento social y económico de Santiago del Estero e incluso de la región al reactivar un antiguo derrotero que conectaba de manera directa a la ciudad mencionada con Jujuy y el Alto Perú, comenzándose desde esos momentos a estrechar relaciones el poder del cabildo con el interés del funcionamiento de una ruta que establecía el itinerario comercial de las primitivas ciudades y en especial de aquellas que se conectaban en forma recta por los llanos.

De acuerdo al análisis representado por el estudio de caso de la familia Alfaro y su red como propietaria de los establecimientos rurales, los precios de las propiedades subieron desde la adquisición de 1714 en \$200 por los derechos de ambas estancias hasta llegar a los $\$ 2.000$ de Tenené en 1731 y sus $\$ 3.000$ en 1753 . Estos precios se encuentran relacionados con los ataques de los Mocovíes, el despoblamiento de la zona y las prohibiciones gubernamentales al Camino del Palomar, además de las reactivaciones por períodos. 
La familia de Alfaro, diagramada estratégicamente con los enlaces de Josefa e Isabel con militares allegados -devenidos luego en comerciantes y políticos-, también tuvo un alcance regional en cuanto a sus actividades mercantiles y agrarias. Los integrantes de la red familiar efectuaron acciones de encomenderos conjuntamente con los intercambios de bastimentos con las carretas que surcaban por el Palomar. Además de esto, participaron en ventas de esclavos a nivel jurisdiccional y en los préstamos locales. Luego de estas situaciones sobrevinieron las ventas de los activos por parte de Isabel, lo que representó el final de un período, la conclusión de una etapa signada por un grupo familiar que desarrolló todas las actividades comerciales conocidas en esa época en Santiago del Estero. La actuación en la red familiar por parte de Isabel representa algo singular para esa época, es decir, la activa participación de una mujer en los negocios de la familia, quien pudo desempeñar un papel económico y social determinante durante la primera mitad de la centuria del 1700.

Gráfico 1: Evolución de precios de las estancias Tenené y Palomar (1714-1828).

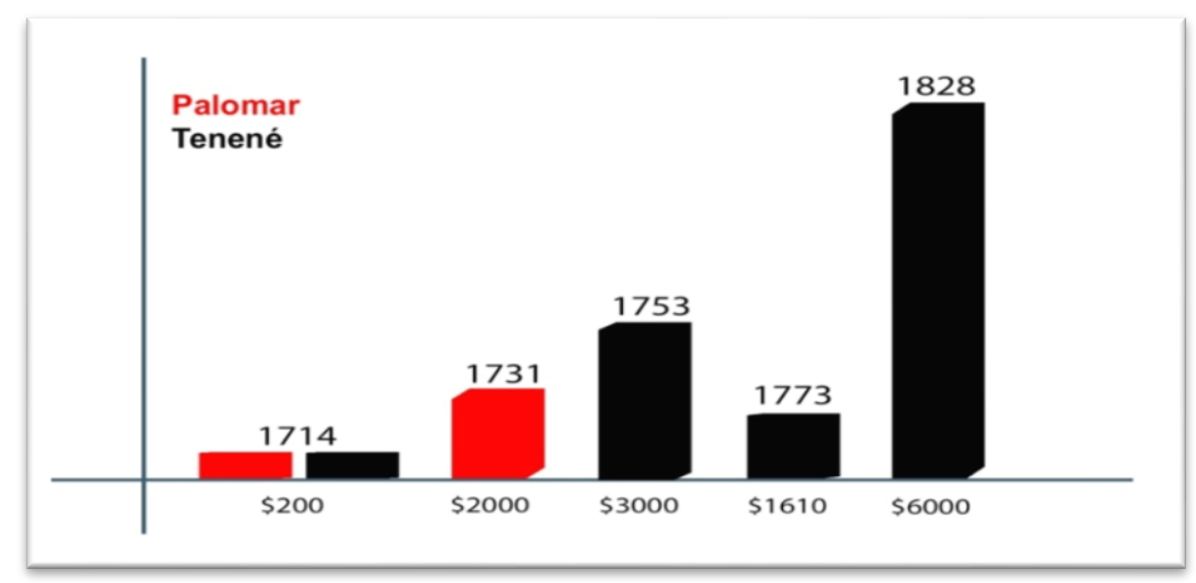

Fuente: Elaboración propia en base a los documentos de la DGCSE.

\section{Notas}

(1) Ver: Peralta Puy, Héctor Francisco. El nacimiento de las ciudades en el norte argentino -con centro en Santiago del Estero- y su correlación con los caminos regionales. Las experiencias en los derroteros coloniales. Siglos XVI, XVII y XVIII, en el Portal www.academia.edu También ver: Peralta Puy, Héctor Francisco. Caminos coloniales en el norte argentino. Elites del Palomar y conflictos por el Camino Real entre Santiago del Estero y Tucumán, Santiago del Estero, Bellas Alas Editorial, 2016.

(2) Ver: Palomeque, Silvia, El Tucumán durante los siglos XVI y XVII. La destrucción de las "Tierras Bajas" en aras de la conquista de las "Tierras Altas"(s/f.), Conferencia presentada durante las VII Jornadas de Investigadores en Arqueología y Etnohistoria del centro-oeste del país. Recuperado de: www.academia.edu;

(3) Para ahondar en la temática referida a las luchas interétnicas desarrolladas por los españoles y los indios en los derroteros y en las fronteras de las ciudades, ver: Peralta Puy, H. F., Caminos coloniales...Op. Cit. 
(4) A medida que fue creciendo su participación política en la región y en el cabildo santiagueño y también su patrimonio material -debido a sus actividades comerciales(comenzando como fletador y luego como mercader tratante), fue necesario para Alfaro consolidar su posición social mediante un matrimonio destacado debido a que la familia que lograba poseer el poder en la representación social podía controlar la función pública conjuntamente con el comercio, por lo que se desposó con doña Manuela de Alba Bravo de Zamora (descendiente de don Lope Bravo de Zamora, quien fuera uno de los conquistadores y primeros pobladores de Santiago del Estero). Esta fue una situación típica en el período colonial debido a los numerosos enlaces realizados entre los peninsulares comerciantes y políticos con las mujeres criollas portadoras de los apellidos tradicionales en el medio local, siendo ésta una de las estrategias para la construcción de las redes familiares dentro de la elite, por lo que se completaron las aspiraciones de Alfaro en cuanto a los vínculos sociales con los nacimientos de Josefa e Isabel y con sus futuros casamientos.

(5) Documento en Archivo Histórico de Santiago del Estero (en adelante AHSE), Tribunales, Legajo 13 Bis, Expediente 47, Año 1700.

(6) Documento en AHSE, Tribunales, Legajo 197, Expediente 68, Año 1702.

(7) Documento en AHSE, Tribunales, Legajo 11 Bis, Expediente 78, Año 1703.

(8) Documento en la Dirección General de Catastro de Santiago del Estero (en adelante DGCSE). Duplicado № 69. Carpeta Tenené, Sección Archivo Gráfico, Duplicado de las operaciones de mensura, deslinde y amojonamiento de la estancia de Tenené y tierras anexas del Pozo Cavado situadas en el Departamento Jiménez. Distritos Pozo Hondo, Yscayacu, Cashico y Vinalito. Propiedad de la sucesión del señor José Manuel Achaval. 1920-1921. Mensura judicial realizada por el agrimensor Francisco David. Fojas 28 y 28 Vuelta.

(9) Para conocer el origen de las estancias y el significado de sus denominaciones, sus representaciones cartográficas, sus actividades económicas y los sucesivos propietarios desde 1640, ver: Peralta Puy, Héctor Francisco, Las inversiones agrarias de la elite santiagueña en el Camino del Palomar. Evolución jurídica y actividades económicas de las estancias y sus propietarios. 1640-1753, XXV Jornadas de Historia Económica. Recuperado de: www.academia.edu. También en Caminos coloniales...Op. Cit.

(10) Esta conceptualización solamente la aplicamos para las estancias Tenené y Palomar, por lo que no debe confundirse su utilización con la ocupación de tierras y establecimientos agrícolas-ganaderos en el resto del territorio santiagueño. Los primeros dos propietarios fueron el Capitán Mateo Bautista Palavecino y la orden de los domínicos residente en los territorios santiagueños.

(11) Este valor se encuentra relacionado con la inestabilidad e inseguridad que había vivido toda esa zona agrícola y fronteriza enmarcada dentro de los caminos comerciales hasta llegar a la inevitable situación de ser despobladas por parte de los religiosos, siendo ésta una de las consecuencias directas emanadas por los avances orientales de los Mocovíes que comenzaron a ser una realidad desde finales del siglo XVII.

(12) Esta reactivación del antiguo Camino del Palomar originó en 1718 la primera denuncia de Tucumán hacia Santiago del Estero por el uso del mencionado derrotero (conflicto finalizado recién en 1788 luego de múltiples enfrentamientos judiciales y personales entre ambos cabildos). Para analizar el inicio, el desarrollo y la finalización del pleito regional, ver: Peralta Puy, H.F., Caminos coloniales en el norte...Op. Cit.

(13) Documento en AHSE, Tribunales, Legajo 8, Expediente 12, Año 1717.

(14) Documento en AHSE, Tribunales, Legajo 11 Bis, Expediente 73, Año 1719.

(15) Documento en AHSE, Tribunales, Legajo 7 Bis, Expediente 148, Año 1750.

(16) Documentos en AHSE, Tribunales, Legajo 11 Bis, Expediente 72, Año 1713 y Legajo 7, Expediente 60, Año 1724.

(17) Documento en AHSE, Tribunales, Legajo 8, Expediente 35, Año 1726.

(18) Documento en AHSE, Tribunales, Legajo 5, Expediente 86, Año 1727.

(19) Documento en AHSE, Tribunales, Legajo 2, Expediente 30, Año 1733. 
(20) Estos créditos usualmente eran adquiridos por animales, efectos de la tierra y de Castilla, y las garantías estaban sustentadas por mercaderías, esclavos o propiedades. Estas cartas de crédito podían poseer un vecino como garante. Por los créditos obtenidos con el presidente de la Real Audiencia de la Plata y con las casas comerciales de Buenos Aires, inferimos que Alfaro tuvo garantías sustentables para contraer esas obligaciones financieras.

(21) En 1711, fue ordenado por Urizar al cabildo santiagueño que tenga preparados a los soldados para el acompañamiento a Alfaro con 512 indios Malbaláes desde el límite de San Miguel hasta Santa Fe y Buenos Aires. Debido a una enfermedad, el encargado de ejecutar la misión por el territorio santiagueño delegó el mando en Peñaloza, quien se puso al frente de los soldados en compañía de Domingo Gerónimo de Frías (eran hombres de confianza de Alfaro quienes contrajeron matrimonios con sus hijas).

(22) Era hijo de Alonso de Frías y Robles y Josefa de Paz y Figueroa, hermana de Juan de Paz y Figueroa el mozo. Este integrante de la elite local fue comandante de Guañagasta, alférez real sustituto y alcalde de segundo voto en 1713. Por los méritos de Frías, Urizar le concedió en 1714 la merced de Tipiro ubicada a 8 leguas hacia el norte de la cuidad. Este territorio fue un pueblo de indios, aunque en el momento del pedido solo quedaba el curaca con su familia. En la solicitud se encuentra una referencia al visitador Luján de Vargas, quien había mandado a que se poblase nuevamente esas tierras con sus ocupantes, aunque los mismos prefirieron quedarse en el pueblo de Pasao (esta era la justificación de Frías para el pedido de las tierras). Además, Frías fue nombrado como administrador del pueblo de indios de Manogasta en 1717 y también tuvo en 1728 la dirección de la recaudación del pueblo de Sumamao, aunque de ello se encargaba su hijo Alonso.

(23) Fue encomendero de Matará (1719), alcalde mayor provincial de la Santa Hermandad (propietario) desde 1727 y teniente de gobernador (1728), entre otras ocupaciones de gobierno.

(24) Documento en AHSE, Tribunales, Legajo 8, Expediente 20, Año 1725.

(25) Documento en AHSE, Tribunales, Legajo 8, Expediente 21, Año 1725.

(26) El Capitán Urrejola fue Alcalde de Segundo Voto en 1733 y 1738 y Alcalde de la Santa Hermandad al año siguiente.

(27) Documento en la Revista del Archivo de Santiago del Estero, Tomo VIII, № 15, Op. Cit., Págs. 31 hasta 35.

(28) Una parte de la riqueza de la red familiar se puede constatar con el monto de la carta dotal que ascendía a $\$ 34.255$, la cual se encontraba compuesta por telas europeas, finos muebles, cinco esclavos y animales que se encontraban en las estancias.

(29) Documento en AHSE, Tribunales, Legajo 7, Expediente 19, Año 1731.

(30) Documento en AHSE, Asuntos Generales, Legajo 2, Expediente 74, Año 1733.

(31) Documento en AHSE, Tribunales, Legajo 9, Expediente 13, Año 1732.

(32) Documento en AHSE, Tribunales, Legajo 7, Expediente 9, Año 1733.

(33) Documento en AHSE, Tribunales, Legajo 7, Expediente 74, Año 1734.

(34) Documento en AHSE, Tribunales, Legajo 9, Expediente 28, Año 1750. Esta operación fue realizada por las actividades desempeñadas por Félix Ignacio en la zona del Salado debido a que era encomendero de Matará (desempeñándose anteriormente su padre Gerónimo), por lo que se explica la entrega de la cera al Cura Párroco de esa Iglesia, Francisco de Luna y Cárdenas.

(35) Documento en la Revista del Archivo de Santiago del Estero, Tomo VIII, N 16, Abril, mayo y junio de 1928, Imprenta Molinari, Santiago del Estero, Págs. 30, 31 y 32.

(36) Documento en Carranza, Ángel Justiniano. Libros Capitulares de Santiago del Estero, 1727-1763, Buenos Aires, Imprenta Europea, 1882, pp. 152 y 153.

(37) Documento en AHSE, Tribunales, Legajo 3 Bis, Expediente 88, Año 1750.

Recibido: julio de 2017.

Aprobado: octubre de 2017. 


\section{Para citar este trabajo}

Peralta Puy, H. "Actividades económicas locales y regionales desarrolladas por las elites en Santiago del Estero. El caso de Alfaro y sus descendientes durante 1700-1750" en Cuadernos de H Ideas [En línea], vol. 11, n 11, diciembre 2017, consultado...; URL: http://perio.unlp.edu.ar/ojs/index.php/cps/article/view/4546 